\title{
Optimization of main steam temperature control system based on improved electromagnetism-like mechanism algorithm
}

\author{
Gui-Quan Xiong ${ }^{1, \mathrm{a}, \dagger}$, Xiao-Yan Wang ${ }^{2, \mathrm{~b}}$ and Dong-Feng Wang ${ }^{\mathrm{c}}$ \\ ${ }^{1}$ Hebei Engineering Research Center of Simulation \& Optimized Control \\ for Power Generation \\ , Baoding, China, 071003 \\ axiongguiquan@163.com, ${ }^{b}$ Wangdongfeng@ncepubd.edu.cn \\ ${ }^{\dagger}$ Corresponding author
}

In this paper, according to a thoughtful study of electromagnetism-like mechanism algorithm, proposing a method of parameters optimization for PID controller based on the improved electromagnetism- like mechanism algorithm. Aiming at these problems that the initial population in the original algorithm is not uniform, the local search efficiency is low, and the resultant force calculation is complex. The algorithm is improved, and it has advantages with fast searching speed and simple principles, which can effectively improve the stability of the control system. Finally, through the simulation of the main steam temperature control system, which is a typical large inertia and large delay thermal object. The PID controller is difficult to meet the performance requirements of the object, the simulation testifies the feasibility and reliability of the algorithm and prominently improves the controlling quality.

Keywords: The main steam temperature control; Electromagnetism-like mechanism algorithm; PID control; Parameter optimization.

\section{Introduction}

The electromagnetism-like mechanism (EM)[1] is a new population-based optimization algorithm, which is proposed by Birbil and Fang. They were inspired by the attraction and repulsion of charged particles in the electromagnetic field. We regard particles as electrons because they posses charge in the algorithm. And function value corresponding is is equal to the charge on particles. Then calculating and analyzing the forces between charged particles according to the Coulomb's law. Finally, calculating space-charge forces for each particle. The direction in which the particles move depends upon the direction of space-charge forces. By simulating iteration operation, it discovers that EM can surely and rapidly get global optimum solution and greatly improve convergence rate. 


\section{The Electromagnetism-Like Mechanism Algorithm}

\subsection{Introduction of electromagnetic field theory}

In 1785, French physicist Coulomb established the famous Coulomb's law [2] through a series of experiments. The main contents include: two static point charges existed in a vacuum, the electrostatic interaction between charged particles is proportional to the product of these two magnitude of charges, and it is inversely proportional to the square of the distance between them. The direction of the force along the line between them, and like charges repel each other, unlike charges attract each other. The vector representation of the Coulomb's law:

$$
F_{12}=k \frac{q_{1} q_{2}}{r_{21}{ }^{2}} \vec{r}_{21}
$$

Among $_{\vec{r}}$ them, $F_{12}$ represents the force of $q_{2}$ to $q_{1}, \mathrm{k}$ is the proportional coefficient, ${ }_{21}$ represents a unit vector in the direction of $q_{2}$ to $q_{1}$, That is the direction of the force between $q_{2}$ and $q_{1}$.It is shown in Fig.1.

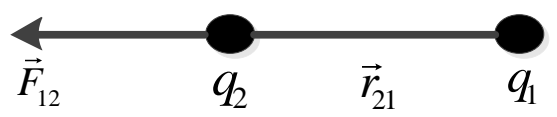

Fig.1 Force between two charges in an electromagnetic field

Whep $q_{1}$ and $q_{2}$ are in the same ${ }_{r_{12}}$ ign, these two charges repel each other, That is $F_{12}$ along the direction of $\vec{r}_{21}$; on the contrary, When $\mathrm{q}_{1}$ and $\mathrm{q}_{2}$ are not in the same sign, these two charges attract each other, That is $F_{12}$ along the direction of $-r_{21}$. The Coulomb's law can be expressed as:

$$
F=\frac{1}{4 \pi \varepsilon_{0}} \frac{q_{1} q_{2}}{r^{2}} \vec{r}=\frac{1}{4 \pi \varepsilon_{0}} \frac{q_{1} q_{2}}{r^{3}} r
$$

\subsection{Optimization analysis of electromagnetism-like mechanism algorithm}

The basic idea of the electromagnetism-like mechanism algorithm is to generate a set of initial solutions in the range of feasible region, and then imitate the attraction and repulsion between the particles in the electromagnetic field. Each particle is considered as a point charge. The magnitude of the charge on each particle is directly related to the function value of the objective function to be optimized. Meanwhile, it also determines the magnitude of the force from one point charge to the other point charges in the whole electronic group. In other words, the smaller the value of the objective function, the greater the attractiveness of the charge. EM algorithm determines the moving direction of 
the particle by calculating resultant force that the other particles apply to the current particle. Finally, the updating rules of the algorithm are proposed to produce the next generation of electrons. Repeating the above iterative process, until all the particles converge to a small neighborhood, after meeting the artificial termination conditions, the optimal solution of the optimization problem is obtained. The flow chart of the EM algorithm is shown in Fig.2

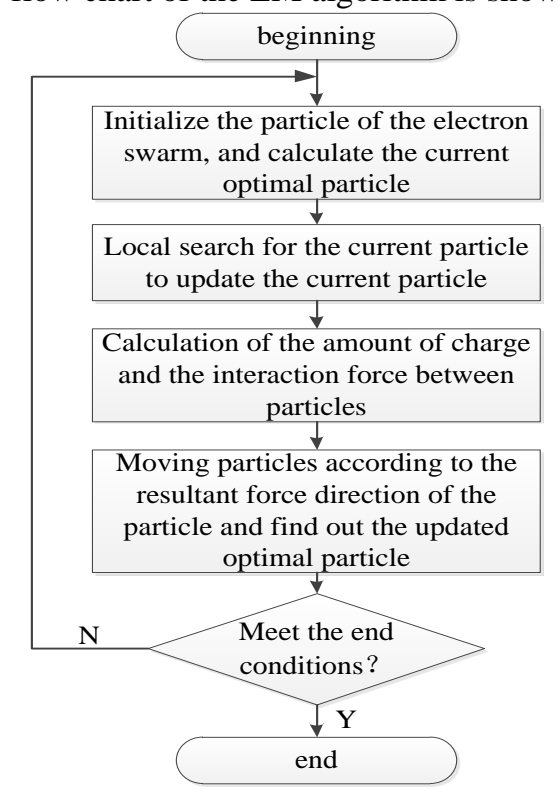

Fig.2 Flow chart of classic EM algorithm

\subsection{Improvement of electromagnetism-like mechanism algorithm}

(1)Using uniform design to construct the initial electron group

Uniform design method [3] based on uniform design table- $U_{m}\left(n^{s}\right), \mathrm{m}$ is the number of experiments, $\mathrm{s}$ is the number of factors, $\mathrm{n}$ is each level of the number of factors. In this paper, we make $m=n$. In practical application, When $m$ is an even number, Uniformity of the uniform design table produced by GLP[4] is poor. So, removing the last line of the uniform design table- $U_{\mathrm{m}+1}\left[(m+1)^{s}\right]$, and the uniform design table- $U_{n}\left(\mathrm{n}^{s}\right)$ is obtained.

$$
a_{i j}=\left(u_{i j}-1 / 2\right) /(m+1), j=1,2,3 \ldots, n
$$

With the above formula, normalizing $u_{i j}$ to interval $1(0,1)$,finally, mapping 
$a_{i j}$ to the initial feasible region of the solution with reference to formula (4).

$$
\begin{aligned}
& x_{i}^{j}=\text { LLimits }_{j}+a_{i j}\left(\text { HLimits }_{j}-\text { LLimits }_{j}\right) \\
& i=1,2 \ldots, m, j=1,2 \ldots, n
\end{aligned}
$$

$x_{\mathrm{i}}^{\mathrm{j}}$ indicates that the initial value of the $\mathrm{j}$ dimension of the $\mathrm{i}$ electron. Among them, HLimits, LLimits are the upper and lower bounds of the optimal parameters.

In this paper, we use stochastic method and uniform design method to generate 100 points in two-dimensional space, they are shown in Fig.3 a and Fig.3 b.

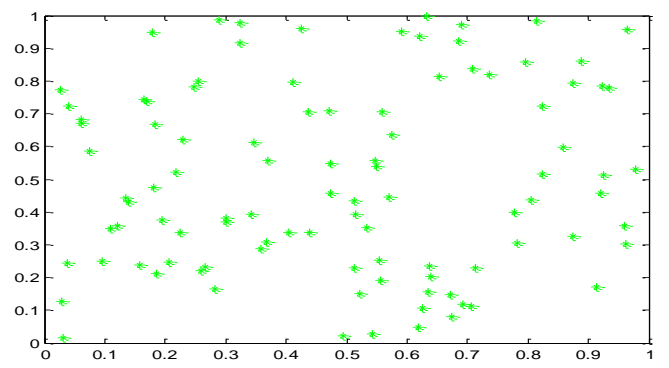

Fig.3 a Randomly generated 100 two-dimensional points

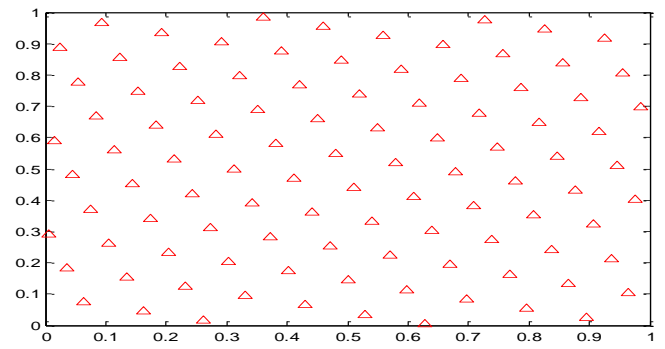

Fig. 3 b Using uniform design table to generate 100 two-dimensional points

(2) The moving step adds correction factor

In the classical algorithm, the moving step of the electron does not take into account the influence of the electronic sub evolutionary algebra on the moving range. Aiming at this problem, this paper proposes the method of using an adaptive mobile operator [4] to control the step size. And an adaptive function is added as follows. 


$$
\phi=\cos \left[\frac{\text { itter }^{\frac{1}{2}}}{6 \text { Maxiter }}\right]
$$

Maxiter is the maximum number of iterations, ${ }^{\text {iter }}$ is the current iteration number, the update rule for the electron:

$$
x_{i+1}=x_{i}+\phi \cdot \lambda \frac{F_{i}}{\left\|F_{i}\right\|} \cdot \text { STEP } ; ;=1, \text { Qn }
$$

Among them, $\lambda \in(0,1), F_{i}$ is the joint force of the electron, in the process of moving, it's feasible moving range is given by the vector as follows:

$\operatorname{STEP}=\left(\xi_{1}, \xi_{2} \ldots, \xi_{n}\right), \xi_{j}(i=1,2, \ldots n)$ indicates that the feasible step for moving the upper boundary and the lower boundary.

\section{Improved Electromagnetism-Like Mechanism Algorithm to Optimize PID Parameters of Main Steam System}

\subsection{Dynamic characteristics analysis of main steam system}

Dynamic characteristics of the main steam temperature is closely related to the running conditions in the whole units. When the load changes, characteristics of unit also changed significantly, the main parameters influenced: main steam flow and main steam pressure and main steam temperature. Among them, the influence of main steam flow (load) is most obvious, mainly reflected in the time constant of inert and guide the static gain changes.

At present the main steam temperature control scheme is often used as shown in Fig.4. $w_{1}(s)$ is the inert area transfer function. ${ }_{2}(s)$ is leading segment transfer function. PI proportional integral controller is adopted in the inner and outer loop.

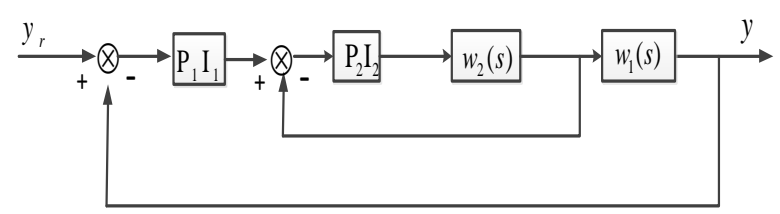

Fig.4 Main steam temperature cascade control system

A 300MW thermal power unit $60 \%$ load, the main steam temperature on the dynamic characteristics of water jet disturbance as shown in the formula (7)[5]: 


$$
w_{1}(s)=\frac{0.523}{(88.5 s+1)^{6}}, w_{2}(s)=\frac{0.93}{(73.3 s+1)^{2}}
$$

\subsection{PID parameter optimization and system simulation based on improved EM algorithm}

PID controller [6] is still the main controller of the industrial field. It's typical transfer function is shown in the formula (8):

$$
u(s)=\frac{1}{\delta}\left(1+\frac{1}{T_{i} s}+\frac{T_{D} s+1}{1+K_{D} T_{D} s}\right)
$$

PID parameters tuning, that is to use a specific mathematical algorithm to optimize the parameters of the PID formula. But its essence is the process of parameter optimization based on a certain objective function. The objective function is the fitness function. The objective function is generally based on the error integral formula [5]. In general, under the unit step disturbance, integral value of a function, which is related to $e(t) e(t)$ is the deviation between given value $r(t)$ and output value $y(t)$. Such as time by absolute error integral criterion (ITAE).

$$
I T A E=\int_{0}^{t_{s}} t|e(t)| d t
$$

We regard the $60 \%$ load as the optimization point, Using improved EM algorithm to optimize the parameters $\left(\delta_{1}, T_{i} 1, \delta_{2}, T_{i} 2\right)$ of PI controllers in inner and outer loop, electron swarm NUMBER_POP $=100$, in this paper, the maximum number of iterations MaxIter=100,Local maximum number of iterations MaxlocalIter=20. The interval of the four parameters to be optimized is :Upper $=[2,300,1100]$, Lower $=[1,180,0.1,50]$

The result of optimization is $\delta_{1=1.1587,{ }_{i} 1}{ }=288.2909,{ }{ }_{2}=0.2276,{ }_{i}{ }^{2}=50.1650$.

When the load of the unit increases, regarding the $75 \%$ load as the optimization point, the dynamic characteristic of the main steam temperature control system is shown in the formula (10)[7]:

$$
w_{1}(s)=\frac{0.7301}{(60.36 s+1)^{6}} \quad w_{2}(s)=\frac{0.544}{(55.64 s+1)^{2}}
$$

The result of optimization is: $\delta_{1}=1.9696, T_{i} 1_{=252.4855}, \delta_{2}=0.2522$, 
$T_{i}^{2}=10.1161$.

When the load of the unit decreases, regarding the $45 \%$ load as the optimization point, the dynamic characteristic of the main steam temperature control system is shown in the formula (11) [7]:

$$
w_{1}(s)=\frac{0.4184}{(106.2 s+1)^{6}} \quad w_{2}(s)=\frac{0.6526}{(76.96 s+1)^{2}}
$$

The result of optimization is: $\delta_{1}=1.2956, T_{i} 1=345.9870, \quad \delta_{2}=0.3181$, $T_{i}^{2}=10.7662$.

After optimization, the unit step response curves are shown in Fig.5,

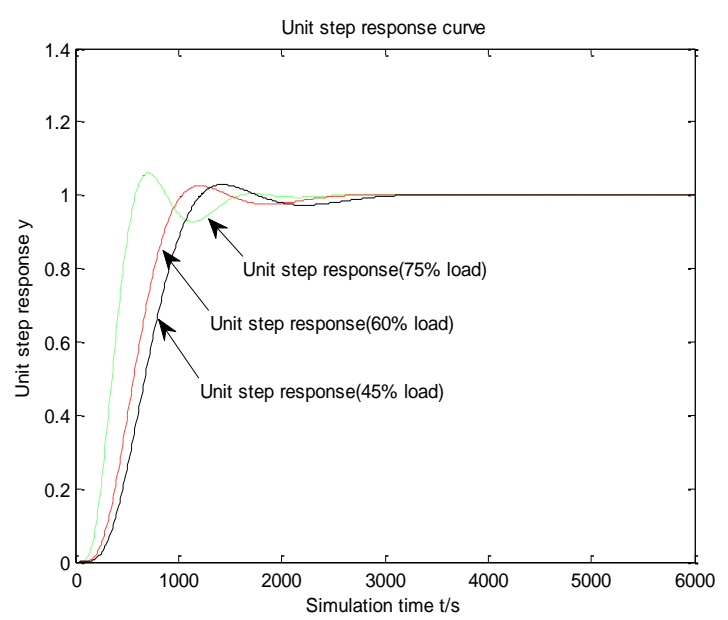

Fig.5 Unit step response curves

The simulating results show that PID parameters tuning is performed based on the improved electromagnetism-like mechanism optimization algorithm can achieve the best control state in the main steam temperature cascade control system. When the load increases and decreases, the system unit step responses indicate that the system has strong robustness.

\section{Summary}

In this paper, the simulating results have shown good control performance, it is demonstrated by digital simulation that the method makes the extra-regulation more small, the regulation time more short, and the robustness better for the temperature control plant.

The EM algorithm is a new global optimization algorithm and it is of 
significance to study the EM algorithm, not only for the global optimization problems with newer and more effective methods, in a certain extent, it promotes the development of science.

Next, the EM algorithm will be applied to the problem of online setting PID parameters and the algorithm will be applied to other areas that is the objective of our further research in this field.

\section{Acknowledgement}

I am very grateful to my tutors Dong-feng Wang who give me a lot of useful advice and help on my thesis. Special thanks should go to my friends and my classmates whose selfless pay and close cooperation let me gain a lot. Finally, I would like to thank my parents and family members for their loving considerations and great confidence in me all through these years.

\section{References}

[1] Yong-qing Liu. Research on electromagnetism-like mechanism algorithm. Xi'an Electronic Science and Technology University, 2013. In Chinese.

[2] Wei-guo Ye. University physics [M]. Beijing: Tsinghua University press, 2012. In Chinese.

[3] Kai-tai Fang. Uniform design and uniform design table [M]. Beijing: Science and Technology Press, 1994. In Chinese.

[4] Guo-qiu Zhang, Wen-xuan Wang. A citation review on the uniform experimental design [J]. Journal of Applied Statistics and Management, 2013, 01: 89-99. In Chinese.

[5] Pu Han, Ze Dong, Dong-feng Wang, Yongjie Zhai, Songming Jiao. Intelligent control theory and application $[\mathrm{M}]$. Beijing: Chinese power press, 2013. In Chinese.

[6] Hui-rong Cao, Li Li. Implementation of uniform design table with MATLAB [J]. statistics and decision, 2008,06:144-146. In Chinese.

[7] Song-ming Jiao, Yu-lin Tan, Shi-jie Sang.PID controller parameters optimization for main steam temperature control system based on improved paticle swarm optimization [J]. Electric Power Science and Engineering, 2012, 12: 9-13. In Chinese.

[8] Chukiat Viwatwongkasem. EM Algorithm for Truncated and Censored Poisson Likelihoods [J]. Procedia Computer Science, 2016, 86.

[9] Yuzhu Tian, Qianqian Zhu, Maozai Tian. Estimation of linear composite quantile regression using EM algorithm [J]. Statistics and Probability Letters, 2016.

[10] Dong-feng Wang, Pu Han. Variable arguments PID control for main steam 
temperature system based on immune genetic optimization [J]. Proceedings of the CSEE,2003, 09: 212-217. In Chinese 